九州大学学術情報リポジトリ

Kyushu University Institutional Repository

\title{
Decomposition and Clustering for the Visualization of Dynamical Systems
}

Arai, Zin

Department of Mathematics, Hokkaido University

ht tp://hdl. hand le. net/2324/1430831

出版情報 : MI lecture note series. 50, pp.104-109，2013-10-21. 九州大学マス・フォア・インダスト リ研究所

バージョン：

権利関係 : 


\title{
Decomposition and clustering for the visualization of dynamical systems
}

\author{
Zin ARAI \\ Department of Mathematics, Hokkaido University
}

\section{INTRODUCTION}

An effective visualization of the global behavior of a dynamical system or a fluid simulation inevitably involves a sort of the partition, or the decomposition of the phase space of the system. This is because, in a generic system there exist uncountably many points having "similar" dynamical behavior and if we plot too many of them then typically we end up with a picture carrying no information. See Figure 1, in which 300 (left) and 3,000 (right) different trajectories of the standard map, the most important example of Hamiltonian dynamics, of length 100 are plotted. It easy to imagine that this problem will be more serious in higher dimensions.
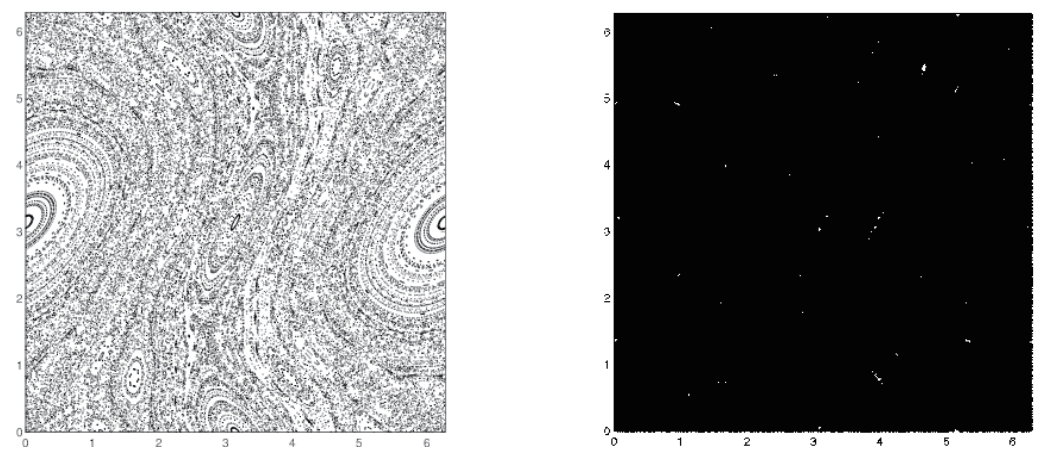

FiguRE 1. Too much information makes no sense

Thus, a natural way to visualize the global behavior of the systems is to classify the points in the phase space into a relatively small number of clusters, so that each cluster corresponds to a particular dynamical behavior.

In following sections, we will explain two ideas for such a clustering. In the next section, we will briefly review the Conley-Morse decomposition, a decomposition of the phase space according to the gradient-like structure of the system. This method does not work fine for conservative dynamics and hence we will discuss another algorithm based on a graph clustering algorithm in the last section. This is a work in progress in the JST CREST project [6]. 


\section{Conley-Morse Decomposition}

In this section, we discuss the method of the Conley-Morse decomposition $[1,2]$. The key idea here is to find small subsets in the phase space which are invariant under the dynamics, and then decompose the phase space into these subsets and connecting orbits among them. These invariant subsets will be called Morse sets.

Historically, this idea was first applied to the gradient flows satisfying a certain non-degeneracy condition by M. Morse. Here by a gradient flow we mean a flow on a manifold $M$ that is defined by the gradient vector field grad $f$ for a smooth function $f: M \rightarrow \mathbb{R}$. Note that in a gradient flow, there will be no chaotic orbit and thus Morse sets are just equilibrium points of the flow. Then C. Conley generalized the theory to arbitrary dynamical systems yielding the celebrated Fundamental theorem of dynamical systems, which says that a dynamical system can always be decomposed into possibly chaotic subinvariant sets (Morse sets) and non-chaotic connecting orbit among them.

Theoretically, there may be infinitely many Morse sets, but since we are mainly interested in the application to practical problems in which noise and errors are inevitably involved, we restrict ourselves to finitely many larger Morse sets. In practice, we fix the grid size for our computation and then ignore Morse sets smaller than the grid size.

Given a dynamical system and a grid decomposition of the phase space, the first step of the algorithm is to define a graph $G$ whose edges imitate the dynamics (see [2]). Then we can expect that a Morse set corresponds to a strongly-connected component in $G$. By collapsing each strongly connected component of $G$ to a single node, we can obtain a much smaller graph representing the structure of the Conley-Morse decomposition (Figure 2). Note that $G$ could be very huge depending on the dimension of the phase space and the size of the grid we are using, however, the graph obtained after collapsing would be much smaller than $G$.

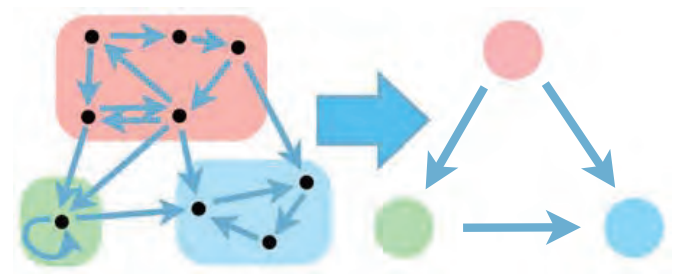

Figure 2. Collapsing strongly connected components of $G$

A node in the collapsed graph corresponds to a Morse set of the system. For each Morse set, we can compute the Conley index, which is an algebraic topological invariant carrying the information of the dynamics in a neighborhood of the Morse set. 


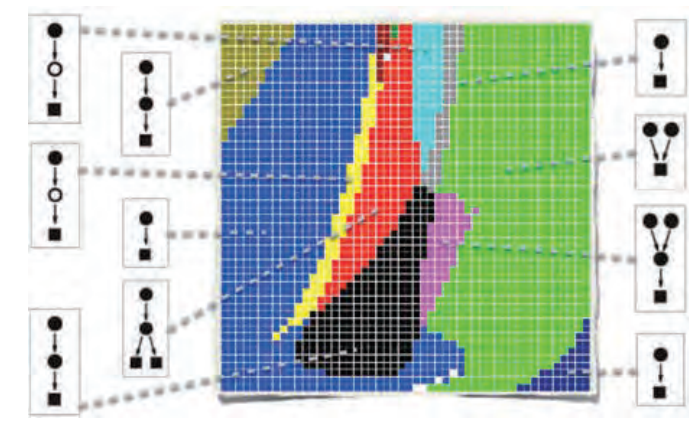

FIGURE 3. the "bifurcation" diagram of the Leslie population model

Figure 3 illustrates an example of the application of Conley-Morse decomposition to the Leslie population model, a map defined by

$$
f\left(x_{1}, x_{2} ; \theta_{1}, \theta_{2}\right)=\left(\left(\theta_{1} x_{1}+\theta_{2} x_{2}\right) \cdot e^{-0.1\left(x_{1}+x_{2}\right)}, 0.7 x_{1}\right) .
$$

The figure shows a decomposition of the parameter $\left(\theta_{1}, \theta_{2}\right)$-plane according to the obtained Conley-Morse graph structure; adjacent boxes in the parameter space with equivalent Conley-Morse graphs are plotted in the same color. A square, a filled circle, a hollow circle in directed graphs indicates an attractor, a Morse set with non-trivial Conley index, and a Morse set with trivial Conley index, respectively.
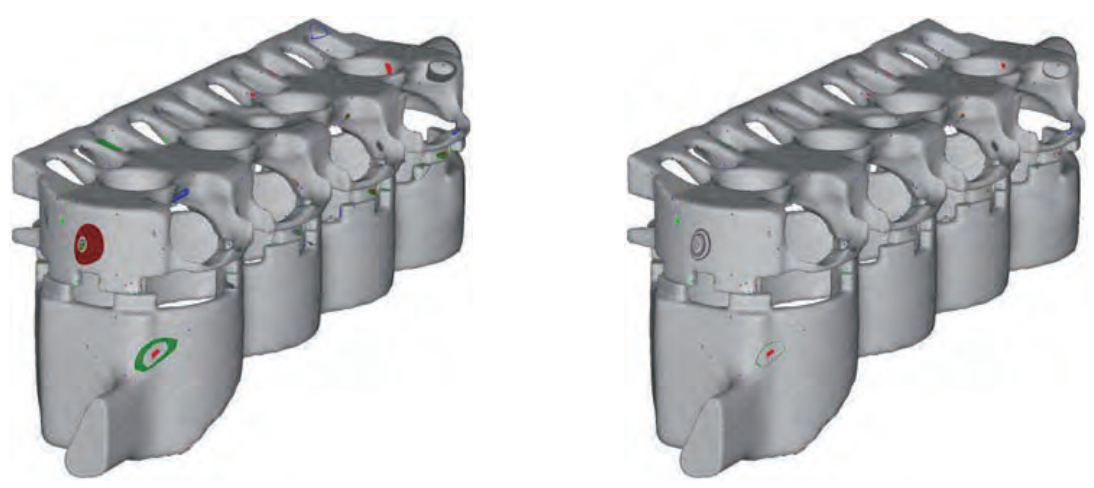

Figure 4. Morse sets for a flow on the surface of a cooling jacket

Although the algorithm works fine for the Leslie model and some other lower dimensional problems, its computational cost is still expensive to be applied to more practical problems. To overcome this computational difficulty, Szymczak et al. developed the method of the piecewise constant approximation of a vector field $[4,5]$ using the theory of differential inclusions. The trajectories in a piecewise constant vector filed 
can be determined by simple geometric rules and hence we can avoid computationally expensive numerical integrations.
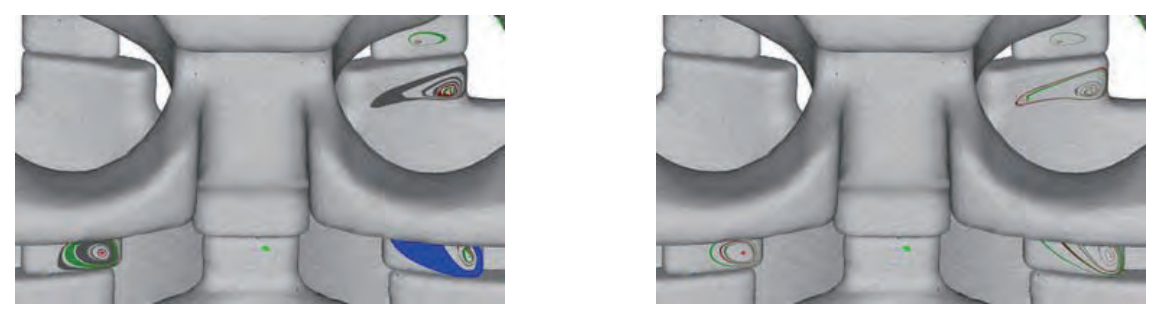

Figure 5. A close up view of Morse sets

Figures 4 and 5 show the result of the application of Szymczak's idea to the vector field on the surface of a cooling jacket which is induced by extrapolating data from 3D fluid simulation in the jacket (figures are provided by the courtesy of A. Szymczak).

\section{Graph Clustering Algorithms}

The idea of Conley-Morse decomposition that we have seen in the previous section works generally well for dissipative dynamics, in which we can expect the existence of attractors and repellers. On the other hand, in conservative systems, for example in Hamiltonian dynamics, there is no attractor and repeller and thus the only possible Conley-Morse decomposition is a trivial one.

To obtain an effective visualization of conservative systems, therefore, another criterion for the phase space partition is required. Note that if the system $T: X \rightarrow X$ is ergodic, we can not use a function $f: X \rightarrow \mathbb{R}$ to find a partition of $X$ because for almost all initial point $x \in X$, the time average $\lim _{n \rightarrow \infty} 1 / n \sum_{i=0}^{i=n} f\left(T^{i}(x)\right)$ takes the same value. This suggests that our criterion should be based on the finite-time behavior of trajectories, rather than the asymptotic behavior as we have done in the Conley-Morse decomposition.

Here we propose a practical algorithm based on a graph clustering algorithm called Peer Pressure Clustering (PPC) [3]. Given a directed graph, PPC iteratively refines an approximated clustering of the graph so that the connectivity inside a cluster will be higher than the connectivity between different clusters. The convergence of the algorithm is not guaranteed for a general graph, however, in most of our cases the algorithm stops after a small number of iterations and runs faster than other clustering algorithms such as Markov clustering.

We apply PPC algorithm for the graph $G$ obtained from a given dynamics (see the previous section). Then the resulting clustering of $G$ corresponds to a partition of the phase space. Since $G$ imitates the behavior of the original dynamics, we can expect that this partition of the phase space enjoys a property similar to the clustering of $G$; 


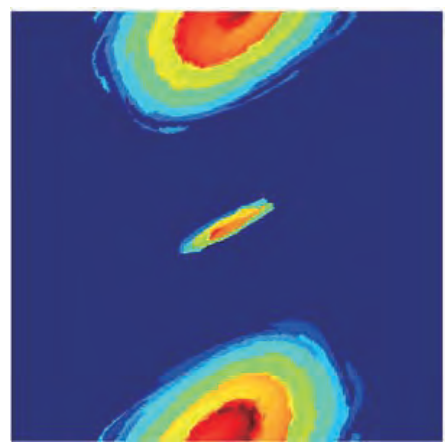

FiguRE 6. PPC algorithm applied for the standard map

the mixing inside a partition element is stronger than then mixing between different partition elements.

Figure 6 shows the result of PPC for a graph obtained from the standard map. We note that quasi-periodic motions and chaotic motions are clearly separated by the algorithm. Figure 7 shows a 2D numerical fluid simulation and the result of PPC applied to it. Two PPC results are shown for different values of a parameter in the algorithm that control the granularity of the clustering.
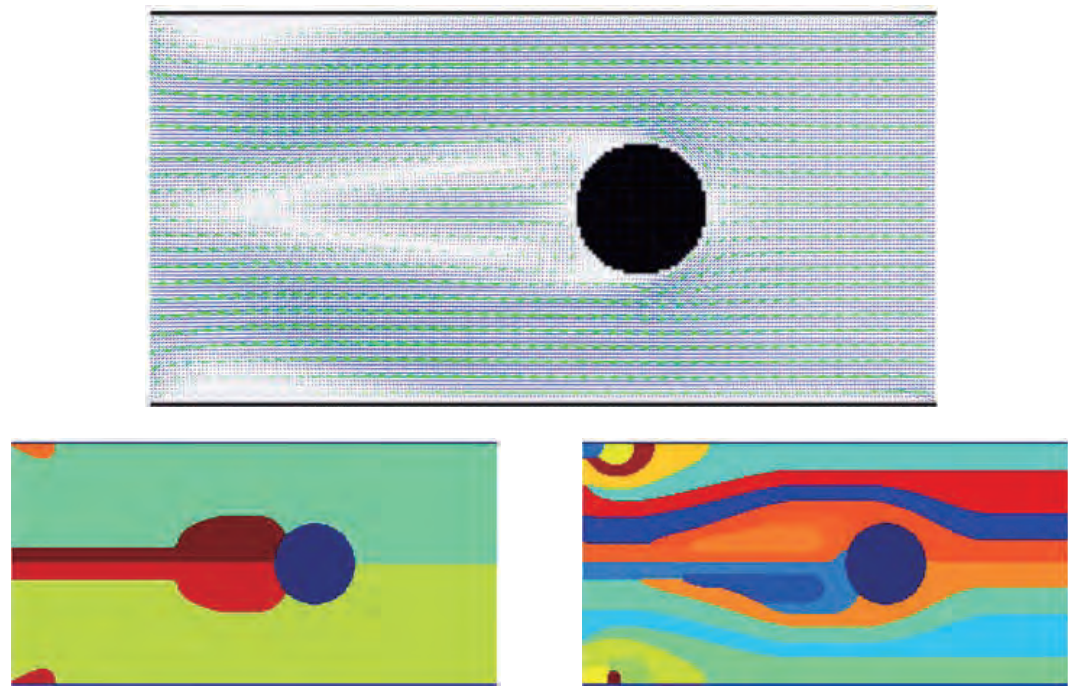

Figure 7. The original flow (top) and the results of coarser (bottom left) and finer (bottom right) PPC clustering 


\section{REFERENCES}

[1] Z. Arai, W. Kalies, H. Kokubu, K. Mischaikow, H. Oka, and P. Pilarczyk: A Database Schema for the Analysis of Global Dynamics of Multiparameter Systems. SIAM Journal on Applied Dynamical Systems, 8(3):757-789, 2009.

[2] Z. Arai, H. Kokubu, and P. Pilarczyk: Recent development in rigorous computational methods in dynamical systems, Japan Journal of Industrial and Applied Mathematics, 26(2-3):393-417, 2009.

[3] J. Kepner and J. Gilbert (editors): Graph algorithms in the language of linear algebra, Society for Industrial and Applied Mathematics, Philadelphia, PA, 2011.

[4] A. Szymczak and E. Zhang: Robust Morse Decompositions of Piecewise Constant Vector Fields, IEEE Transactions on Visualization and Computer Graphics, 18(6), 938-951, 2012.

[5] A. Szymczak: Morse connection graphs for piecewise constant vector fields on surfaces, Computer Aided Geometric Design, 30(6):529-541, 2013.

[6] The CREST project "Toward a paradigm shift created by mathematics of vortex-boundary interactions", Web Page: http://www.math.sci.hokudai.ac.jp/crest/ 